\title{
A KIND OF SELF_TUNING PID CONTROLLER
}

\author{
LE BA DUNG \\ Institute of Information Technology
}

\begin{abstract}
Summary. A Self-tuning PID controller (STC) using a linguistic description of the control strategy and based on the theory of fuzzy set is presented in this paper.
\end{abstract}

\section{INTRODUCTION}

Despite the advent at many sophisticated control theories and techniques, the majority industrial process nowadays are still regulated PID controller. The PID controller and especially the self_tuning PID controller are in full play of control systems. For this reason we can introduce some kinds of them. Firstly, the self_tuning PID is designed based on Ziegler_Nichols princip (1942) and secondly, on other princip, such as

- STC by ultimate Sensitivity method

- STC by limit cycle method

- STC by least square method

- STC by pattern recognite method

- STC by Neuron Net method

In this work the STC by expert method is presented. There are two types of STC using expert system

1. To correct PID parameters according to the response ware patterns

2. To correct PID parameters using the fuzzy control

\section{PROBLEM}

F

The main ideas in this work are concerned with closed_loops response (see Fig 1). The stategy to tune the parameters of the PID controller is based on I, II, III, IV stage of closed Joops response. At the stages I and III the output of the system approaches to the setpoint. To accelerate the system response at these stages are needed. On the contrary at the stages II, IV the output derivates away from the setpoint, the system response must be slowdown. 


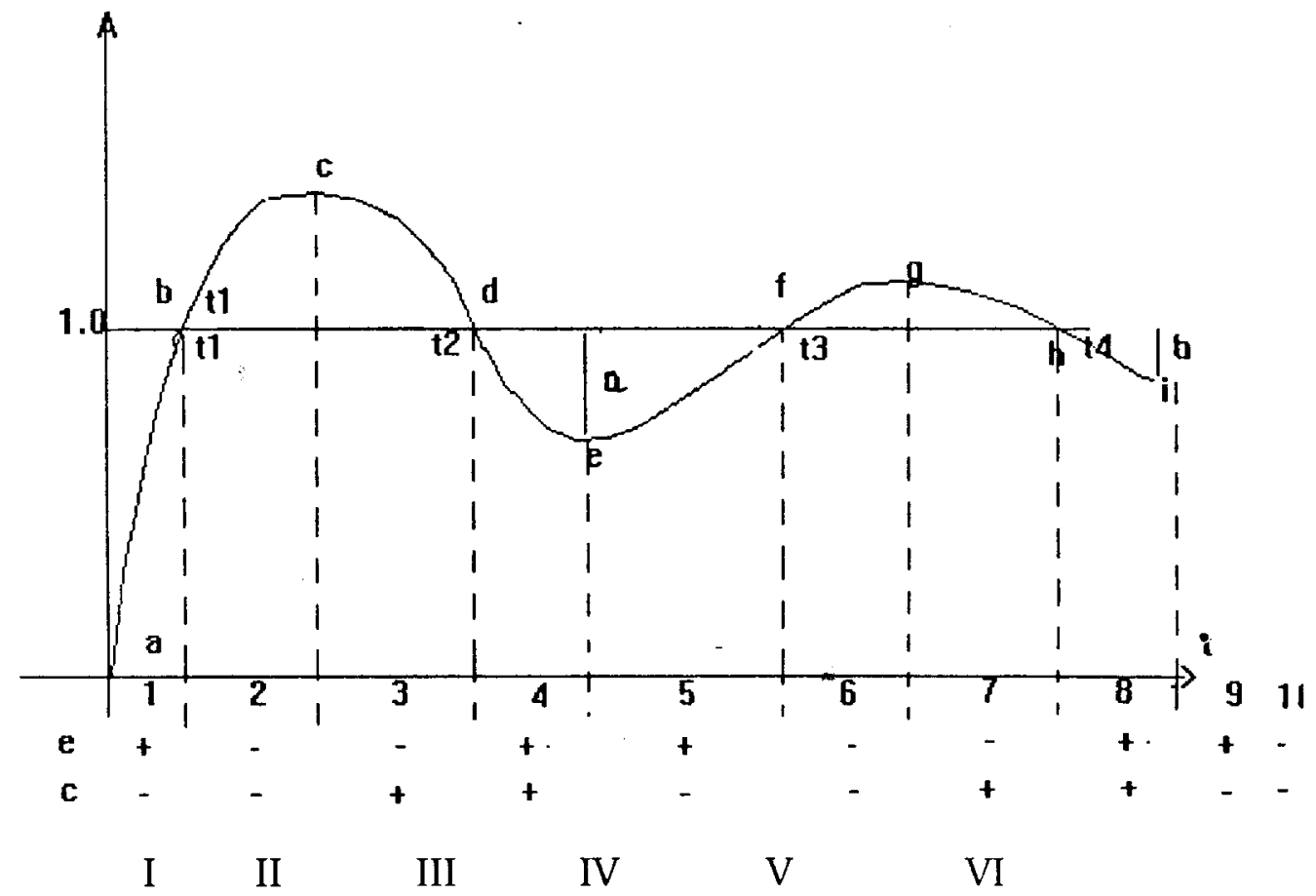

Fig 1. Typical closed_loops response of control systems

\section{THE SELF - TUNING PID CONTROLER}

Basic structure

In this paper we asume that the process to be controlled has single input $u(t)$ and single output $\mathrm{y}(\mathrm{t})$. The STC PID controller must be to bring the process output $y(t)$ to prescribed setpoint $r(t)$. Fig 2 is the block scheme of STC PID controller. The closed_loops error $e(t)=r(t)-y(t)$ and the control $u(t)$ has the following standard form in discret time $k$

$$
u(k)=K\left\{e(k)+\frac{T}{T_{i}} \sum e(k)+\frac{T_{d}}{T}\{e(k)-e(k-1)]\right\}
$$

or in continuouse time $t$

$$
u(k)=K p\left(e(t)+K d(d e(t) / d t)+K_{i} \int e(t) d t\right.
$$

where

$u(k)$ control at the time $k$

$e(k)$ error at the time $k$ 
$T_{i}$ The integral time

$T_{d}$ derivative time

$K$ proportial gain

$T$ sampling periode

The $K, T_{d}, T_{i}$ or $\left(K_{p}, K_{i}, K_{d}\right)$ are the constants of the controller which are to be adjusted on line.

On_line adaptation of the fuzzy control rules

At the Fig 1. the envelope of the peak values of the positive or negative values can be written

$$
y_{b}=1 \pm \frac{e^{-\xi \alpha n t}}{\sqrt{1-\xi^{2}}}
$$

where

$n$ undamped natural frequency,

$\xi$ equivalent damping ratio,

$\omega=\omega n \sqrt{1-\xi^{2}}$ damped frequency.

The approximately of the sinusoid envelope with (Fig 1)

$$
\omega(t 3-t 1)=\omega(t 4-t 2)=2 \pi
$$

Then it can to see the ratio of the two positive (negative) peaks of closed_loops response

$$
\frac{b}{a}=e^{-2 \pi \xi \sqrt{1-\xi^{2}}}=e^{-2 \pi \Delta}
$$

where

$$
\Delta=\frac{\xi}{\sqrt{1-\xi^{2}}}
$$

From Fig 1 the damped period of oscilation $P$ is given by

$$
P=(t 3-t 1)
$$

Then the ultimate period $P u$ is found

$$
P u=\frac{P}{\sqrt{1+\Delta^{2}}}
$$


Because

$$
\sqrt{1+\Delta^{2}}=\sqrt{1+\frac{\xi^{2}}{1-\xi^{2}}}=\frac{1}{\sqrt{1-\xi^{2}}} .
$$

According to the Ziegler_Nichols the proportional gain is

$$
K p=0.5 K u
$$

On the other hand The ultimate gain $K u$ is a function of $K p$ and $\Delta$. From (8) we can expansion for ratio $K u / K p$

$$
K u / K p=\alpha_{0}+\alpha_{1} \Delta+\alpha_{2} \Delta^{2}+\ldots
$$

If $K p=K u$, then $\Delta=0$ and $\alpha_{0}=1$.

The initial value of $K p$ can be written in the form

$$
K p_{0}=K u /\left(1+\alpha_{1} \Delta^{*}\right)
$$

From (9) the values of $\alpha_{1}$ can be be calculated. By virtue of Ziegler - Nichols formula (1942) with $b: a=1: 4$ we can to get $\Delta^{*}$ from (8). From equation $K p=K u /\left(1+\alpha_{1} \Delta^{*}\right)$, with $b: a=1: 4$ we get $\Delta^{*}=0.2207$. Then

$$
\alpha_{1}=4.5310
$$

From (9), (10), (11) we can get

$$
K p_{0}=\left[1+4.5310 /\left(1+4.5310 \Delta^{*}\right] K p\right.
$$

With $\Delta^{*}=0.3$

$$
K p(k+1)=(0.423+1.784 \Delta) K p(k)
$$

From (5), when $\Delta^{*}=0.5$, i.e. $b: a=1: 22$ then

$$
K p(k+1)=(0.3+1.4 \Delta) K p(k) .
$$

From (1) we can get the parameters of PID by following Ziegler-Nichols formula

$$
\begin{aligned}
K i & =\frac{2 \sqrt{1+\Delta^{2}}}{p} \\
K d & =\frac{P}{8 \sqrt{1+\Delta^{2}}} .
\end{aligned}
$$


We can get from Fig 1 with $(t 3-t 1)=2 \pi$, when $0<<2-t 1$ we have

$$
d\left(t_{3}+\tau\right)=\exp (-2 \pi \Delta) d\left(t_{1}+\tau\right) .
$$

It is easy to see

$$
\exp (-2 \pi \Delta) \int_{t_{1}}^{t_{2}} e(t) d t=\int_{t_{3}}^{t_{4}} e(t) d t
$$

Equation (17) points out that $\Delta$ is function of $e(k)$, then we can write

$$
\Delta=F(e(k))
$$

From equation (18) can calculate $\Delta$. According to the Fig 1. at the stage I, III, when the $y(t)$ approaches to the set_point $r(t)$, the $\Delta$ of (14), (15), (16) will be increased, then increasing $K p, K i$, and decreasing $K d$. Siminarly, when $y(t)$ deverges up/down from set point $r(t)$ at the stages II, IV, the $\Delta$ of (14), (15), (16) will be decreased, then decreasing $K p, K i$ and increasing $K d$ consequently. The devergence of the output $y(t)$ will be slowed down. The parameter adjustment of PID controller is proposed by

$$
\Delta(k)=\Delta(k-1)-e(k-1)(\{e(k-1)-e(k-2)\} / T) \beta,
$$

where:

$T$ sampling period

$\Delta($.$) the above description$

$e($.$) the above description$

$[e(k-1)-e(k-2)] / T$ rate of change in errors of last time

$e(k-1) c(k-1)$ the sign of tha stages I, II, III, IV

$\beta$ parameter of the fuzzy inference

The calculation of fuzzy parameter

The calculation of the $\beta$ is based on

1. The basic method $[1]$

2. A mathematical model and algorithm of fuzzy controller [3].

The basic of this calculation is described on the hand:

$$
\text { If } e \text { is } E_{i} \text { and } c \text { is } C_{j} \text {, then } \beta \text { is } \beta_{i j}
$$


where $I, J$ denote the index sets, $E_{i}, C_{j}$ and $\beta_{i j}$ denote liguistic values (fuzzy sets) of $E, C, \beta$, respectivly, with the membership functions

$$
\mu E_{i}(x): X \rightarrow[0,1] ; \mu C_{j}(y): Y \rightarrow[0,1] ; \mu(z): Z \rightarrow[0,1] .
$$

Each rule (20) may be written as a fuzzy implication

5

$$
E_{i} \rightarrow C_{j} \rightarrow \beta_{i j}, \text { where } i \in I, j \in J .
$$

Formula (20) gives a fuzzy relation $R$ on the space $X \times Y=Z$ with the membership function

$$
\mu R_{i j}(x, y, z)=\min \left(E_{i}(x), \mu C_{j}(y), \beta_{i j}(z)\right)
$$

and

$$
R=\max _{i j} R_{i j}
$$

Therefore every fuzzy value $x$ and $y$ are the observation on $E^{\prime}, C^{\prime}$, resperctivly, then fuzzy control $\beta$ is calculated according to the compositional rule of inference

$$
\beta^{\prime}=\left(E^{\prime} \times C^{\prime}\right) \circ R
$$

Formula (24) has a form

$$
\mu \beta^{\prime}(z)=\max _{x, y}\left(\min \left(E^{\prime}(x), C^{\prime}(y), R(x, y, z)\right)\right) .
$$

In the other hand, from 2 based on [3] and 1. (A mathematical model and algorithm of fuzzy controller) we can write that the output of fuzzy inference is described by

$$
F\left(E^{\prime}, C^{\prime}\right)(z)=\max _{x y}\left(\min \left(E^{\prime}(x), C^{\prime}(y), R(x, y, z)\right)\right)
$$

where

$E, C$, are the normal classes of convex fuzzy set

$F\left(E^{\prime}, C^{\prime}\right)$ the ouput of fuzzy inference

$E^{\prime}, C^{\prime}$ are convex fuzzy set on the observation

The existence of fuzzy relation $R$ is proved in [3]. We can see the fuzzy set of $E$ and $C$ below

$$
\begin{aligned}
& E=\{B, N M, N S, Z O, P S, P M, P B\} \\
& C=\{N B, N M, N S, Z O, P S, P M, P B\} \\
& \beta=\{N B, N M, N S, Z O, P S, P M, P B\} .
\end{aligned}
$$

Fig 3 The block scheme of Simulation of STC PID 
8

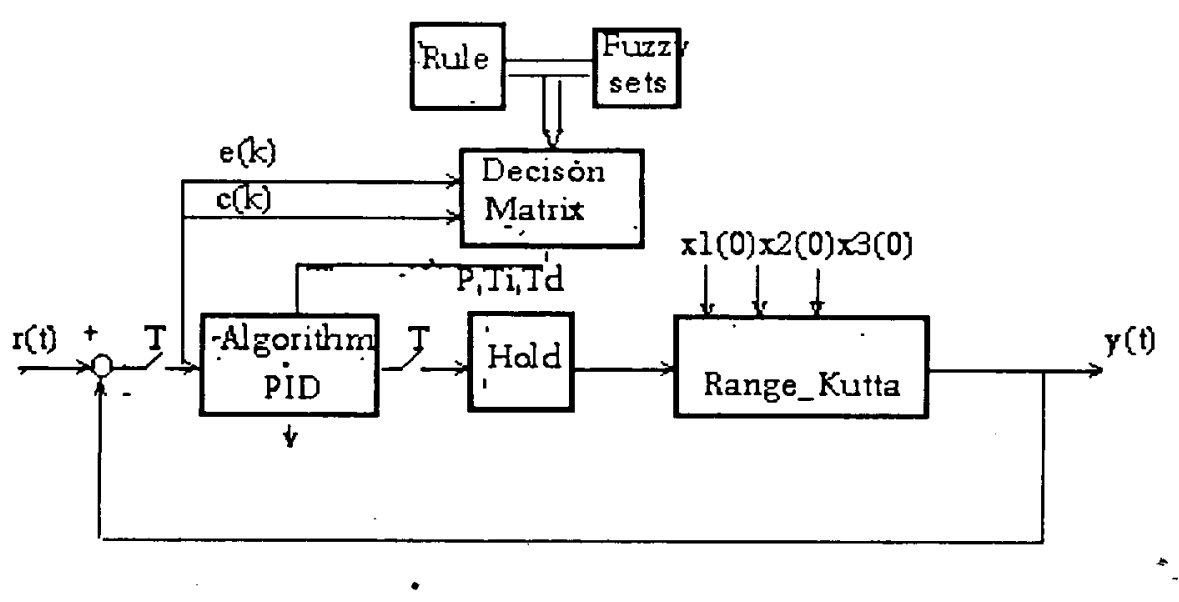

Fig.3 Simulation of STC-PID on PC by language C

\section{CONCLUSION}

This paper proposes STC - PID. The model of STC - PID controller is based on two concepts: the inference of STC -PID is described by Generalized Moduses ponens method and the mathematical model of fuzzy controller. The simulation is proved and the output of control system is approched to the setpoint, despite the parameters of controled system are varied in wide range *s

\section{REFERENCES}

1. Zimmemmann H.J., Fuzzy set theory and applications, Klumer Nijhoff publ. 1985.

2. Yamamoto S., Industrial developments in intelligent and adaptive control, IFACITAC Singapore 1991.

3. Song $\mathrm{Da} \mathrm{He} \mathrm{A}$ mathematical model and algorithm of fuzzy controller, Applcation of fuzzy methodologies in industrial engeneering, Amstredam 1989.

4. Chidambara M.R., A new techniques for tuning controller, Chemical engeneering, 1973. 